\title{
Benign Fibrous Histiocytoma of the Sacrum: A Rare Scenario
}

\author{
Ashish Acharya ${ }^{1, \odot}$ Sarvpreet Singh Grewal ${ }^{1, \odot}$ Paul Sudhakar John $B^{1, \odot}$ \\ ${ }^{1}$ Department of Neurosurgery, Christian Medical College, Ludhiana, \\ Punjab, India \\ Address for correspondence Ashish Acharya, MCh, Department of \\ Neurosurgery, Christian Medical College, Ludhiana, Punjab 141008, \\ India (e-mail: ashish.acharya@live.in).
}

Indian J Neurosurg 2022;11:70-72

\begin{abstract}
Keywords

- sacral tumor

$-\mathrm{BFH}$

- spine tumor

Benign fibrous histiocytoma (BFH) of bone is quite rare, and here we report the second case of such tumor originating from sacrum, with the first being reported in an 18-year-old female. The overlapping clinical, radiological, and histopathological findings make it a difficult diagnosis along with the fact that it is a rare diagnosis. In this case report, we present the diagnostic difficulties and optimal treatment for such cases. A 46-year-old male w presented to OPD with complaint of numbness in left foot since 1 month, and intermittent urinary incontinence for 2 weeks. On examination, the straight leg raising test was positive of left side, extensor hallucis longus (EHL) was weak in both lower limbs, and bilateral ankle jerks were diminished. MRI showed well-defined lobulated solid mass lesion, which is T1 hypointense and T2 hyperintense and homogeneously involving the S1 vertebral body, with involvement of the right sacral ala, and right sacral foramen seen at S1 and S2 levels extending into the spinal canal till $\mathrm{L} 4$ level. Excision through a posterior midline incision was performed taking care to preserve the traversing nerves. Postoperatively, remarkable improvement in pain with no major residual neurological deficit was observed. Patient was followed-up till 9 months; patient's incontinence improved over a period of 3 months and has stayed the same until the last follow-up.
\end{abstract}

\section{Introduction}

Benign fibrous histiocytoma (BFH) of the bone is an interesting and rare $^{1-4}$ tumor. The first case of BFH sacrum was reported in an 18-year-old female patient. ${ }^{5}$ We present here the second case of BFH arising from sacrum. BFH is locally aggressive and has to be differentiated from other benign fibrous bone lesions like xanthomas, nonossifying fibroma, and fibrous cortical defect.

\section{Case History}

A 46-year-old male came to OPD with numbness of left foot for 1 month, developed severe low backache intermittently,

published online

November 24, 2021
DOI https://doi.org/

10.1055/s-0041-1726605 ISSN 2277-954X and was unable to ambulate during such episodes. He also had intermittent urinary incontinence for last 2 weeks. Examination revealed numbness over medial aspect of left foot and left hallux, and bilateral ankle jerks were diminished. Perianal sensations were normal, with preserved anal reflex. MRI showed well-defined lobulated solid mass lesion, which was T1 hypointense and T2 hyperintense and homogeneously involving the S1 vertebral body, with involvement of the right sacral ala, and right sacral foramen seen at S1 and S2 levels extending into the spinal canal till L4 level (-Fig. 1).

Histopathological findings: ( - Fig $2 \mathrm{~A}$ shows plump to spindled tumor cells with hyperchromatic nuclei, H\&E 400x. B-D) Immunohistochemistry showed positivity for EMA,

(C2021. Neurological Surgeons' Society of India.

This is an open access article published by Thieme under the terms of the Creative Commons Attribution-NonDerivative-NonCommercial-License, permitting copying and reproduction so long as the original work is given appropriate credit. Contents may not be used for commercial purposes, or adapted, remixed, transformed or built upon. (https://creativecommons.org/licenses/by-nc-nd/4.0/).

Thieme Medical and Scientific Publishers Pvt. Ltd. A-12, 2nd Floor, Sector 2, Noida-201301 UP, India 

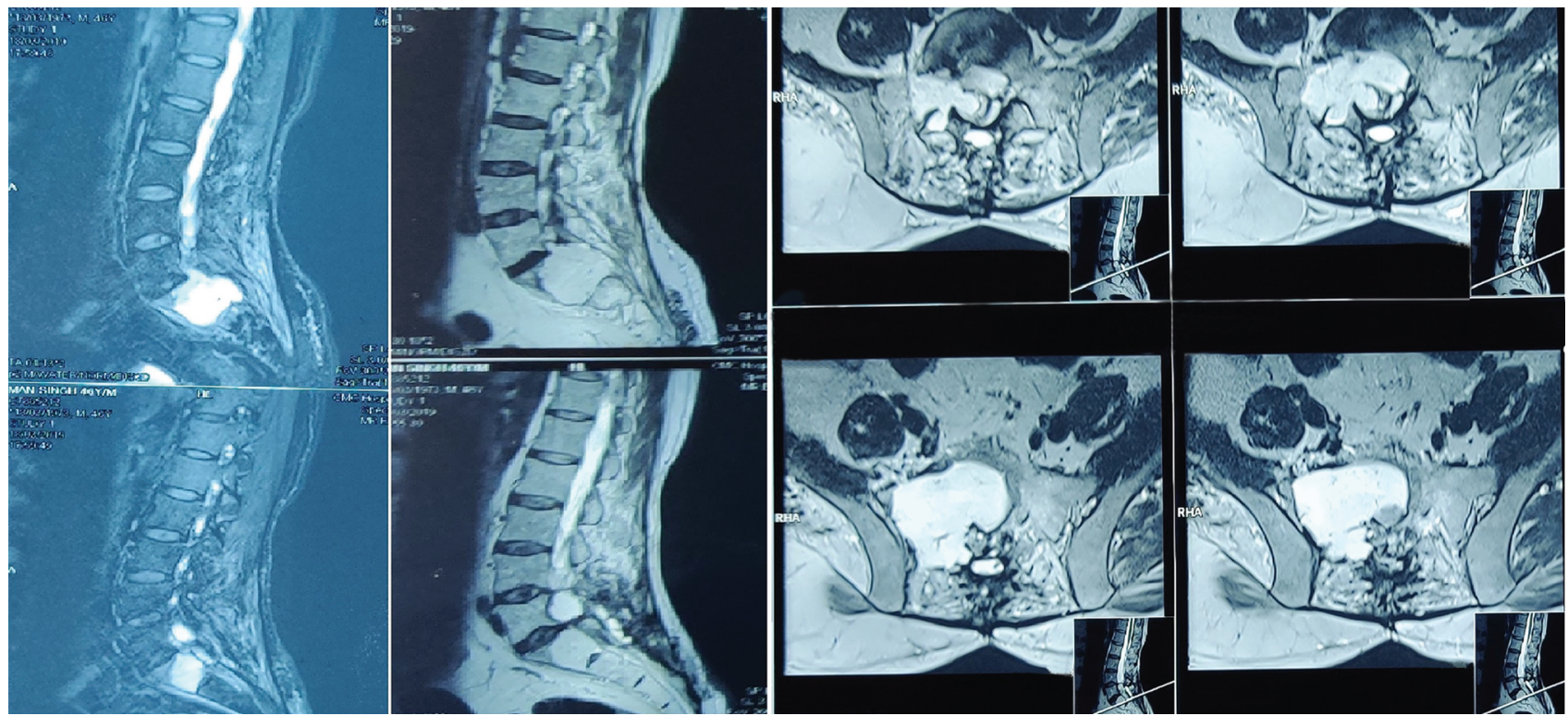

Fig. 1 MRI showing saggital T2 and axial T1 images. The findings have been described in text.

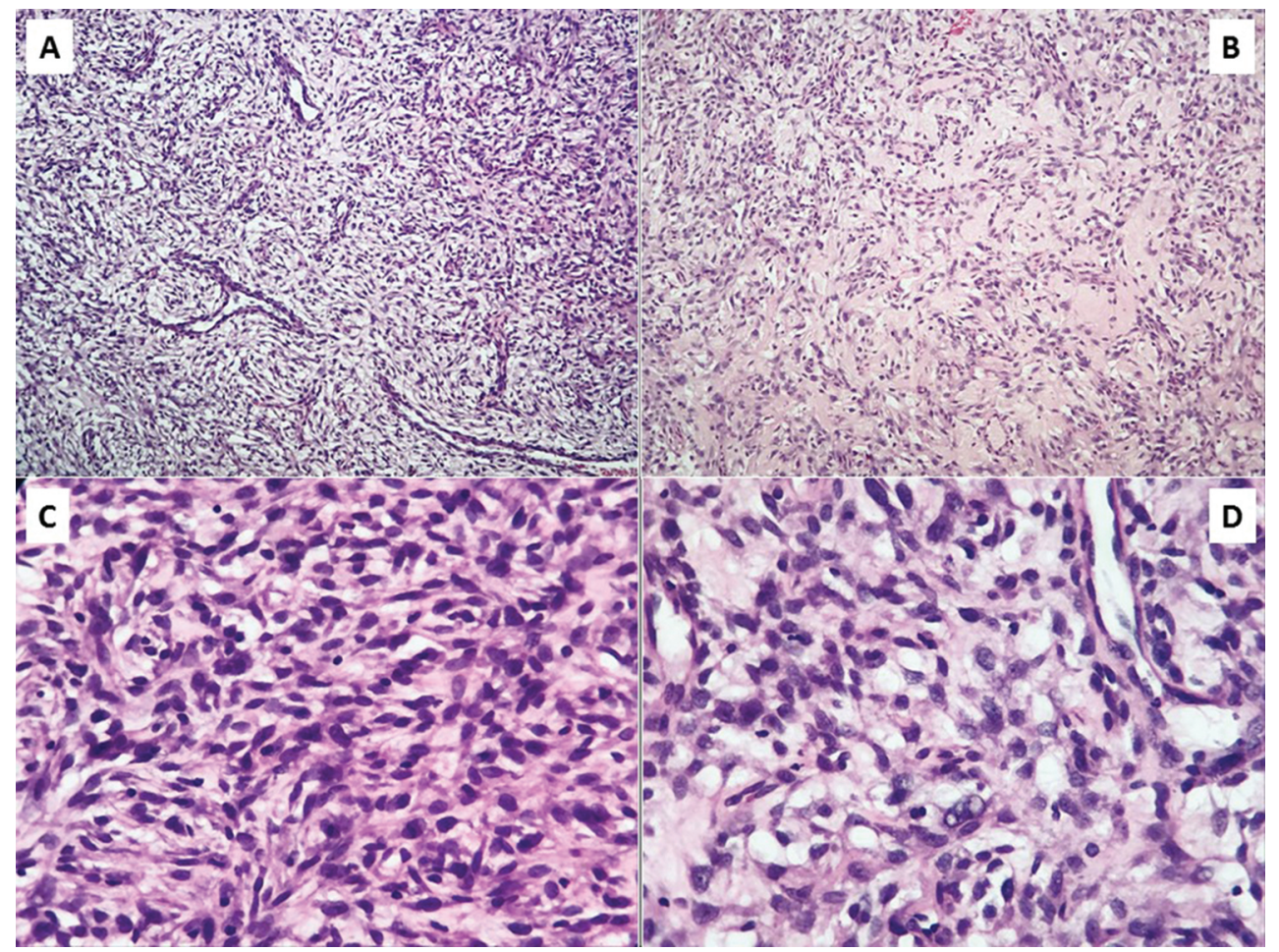

Fig. 2 Histopathological findings: (A) showing plump to spindled tumor cells with hyperchromatic nuclei, H\&E 400x. (B-D) Immunohistochemistry showed positivity for EMA.

S-100 and CK. Tumor cells show round to oval, moderately pleomorphic nuclei with clumped chromatin and inconspicuous nucleoli; scattered infiltrate of chronic inflammatory cells seen, H\&E 400x.

A final diagnosis of BFH of the sacrum was made.

Exploration was done via dorsal midline incision and bilateral laminectomy of S1, 2, and L5 was performed. Tumor was pale, rubbery, and moderately vascular. Care was taken to preserve all the cauda equina structures. Utmost precautions were taken to stay posterior to presacral fascia. Postoperatively, the patient developed cerebrospinal fluid (CSF) leak which was managed conservatively and stopped by 7 days. The patient was sent home with bladder training and pelvic floor strengthening exercises. At 9 months of follow-up, the patient was symptomatically improved with improved bladder control and improved extensor hallucis 
longus (EHL) weakness. Patient was ambulatory and his backache had significantly diminished.

\section{Discussion}

In general, very few cases of BFH arising from other bone sites have been reported. ${ }^{1-7}$ It is very interesting to see and report this as the second case of BFH of sacrum with canal stenosis and neurological signs and symptoms of presentation.

The various primary sacral tumors described in their descending order of frequency are giant cell tumor, chordoma, aneurysmal bone tumor, osteoblastoma, osteosarcoma, and chondrosarcoma. ${ }^{8}$ Malignant fibrous histiocytoma, leiomyosarcoma, and myxopapillary ependymoma occurring outside the central nervous system (CNS) are other rare primary sacral tumors. ${ }^{8}$

$\mathrm{BFH}$, classified as fibrohistiocytic groups of soft-tissue tumors, is still poorly understood since its inception 30 years ago. ${ }^{9}$ The histopathogenesis of the malignant form of fibrous histiocytoma ${ }^{5}$ helps in the understanding of fibrous histiocytoma. Ultrastructural, enzyme histochemical, and immunohistochemical studies show similarities to the "fibro histiocytoid" cell lines of chronic inflammation. ${ }^{9}$ The salient features are prominent storiform pattern of uniform spindle cells with ill-defined eosinophilic cytoplasm and bland, scattered lymphocytes, either multinucleated giant cells, osteoclastic giant cells or foam cells in 59\%, usually less than 5 mitotic figures/10 HPF and the myxoid or hyaline stroma.

The first difficulty involves differentiating this lesion from a soft-tissue lesion and nonossifying fibroma. Criteria ${ }^{5,9}$ are used to define this lesion as arising from the bone, because radiologically it is centred over the bone; it does not breach the presacral fascia anteriorly and is confined to the bone posteriorly. Nonossifying fibroma, although histologically similar to BFH, is primarily found primarily in children of 7 to 13 years of age, found in growing bones, remains asymptomatic for a long time, disappears spontaneously, and is discovered incidentally.

$\mathrm{BFH}$ is locally aggressive and presents with pain due to either pathological fracture or neurogenic in nature. ${ }^{5,9}$ Additionally, other related benign fibrous tumors also need to be differentiated. This is supported by a similar case report of myxoma progressing to myxofibrosarcoma in a young woman, which was found to be recurring five times before the final diagnosis was made., ${ }^{5,7}$ Radiologically, chordoma and $\mathrm{BFH}$ are homogenous and isointense without variable contrast enhancement, while aneurysm bone cyst might have fluid levels, and giant cell tumor is heterogenous with irregular contrast enhancement. While BFHs are known to be locally aggressive, data regarding their malignant transformation are scarce. There has been one instance when a malignant transformation occurred in an atypical fibrous histiocytoma. ${ }^{5,9}$

$\mathrm{BFH}$ of the sacrum has many differential diagnoses, and it is imperative to arrive at the right diagnosis in wake of its rarity at this site. Although local treatment seems adequate (with meticulous techniques to preserve the nerve roots and pelvic stabilization), it is of outmost important to keep these patients on regular follow-up to diagnose any malignant transformation or recurrence.

\section{Conflict of Interest}

None declared.

\section{References}

1 Bertoni F, Calderoni P, Bacchini P, et al. Benign fibrous histiocytoma of bone. J Bone Joint Surg Am 1986;68(8):1225-1230

2 Ceroni D, Dayer R, De Coulon G, Kaelin A. Benign fibrous histiocytoma of bone in a paediatric population: a report of 6 cases. Musculoskelet Surg 2011;95(2):107-114

3 Dahlin DC, General aspects and data on 6,221 cases. In: Bone Tumors. 3rd ed. Springfield: Charles C Thomas; 1978:116-36

4 Demiralp B, Kose O, Oguz E, Sanal T, Ozcan A, Sehirlioglu A. Benign fibrous histiocytoma of the lumbar vertebrae. Skeletal Radiol 2009;38(2):187-191

5 Fletcher CDM, McKee PH, Soft tissue tumours. In: McGee JOD, Isaacson PG, Wright NA, eds. Oxford Textbook of Pathology. New York: Oxford University Press; 1992

6 Balasubramanian C, Rajaraman G, Singh CS, Baliga DK. Benign fibrous histiocytoma of the sacrum-diagnostic difficulties facing this rare bone tumor. Pediatr Neurosurg 2005; 41(5):253-257

7 Brennan MF, Alektiar KM, Maki RG, Sarcomas of the soft tissue and bone. In: Devita VT, Hellman S, Rosenberg SA, eds. Cancer, Principles and Practice of Oncology. 6th ed. Philadelphia: Lippincott Williams \& Wilkins; 2001

8 Imai Y, Yamakawa M, Sato T, Suda A. Malignant fibrous histiocytoma: similarities to the "fibrohistiocytoid cells" in chronic inflammation. Virchows Arch A Pathol Anat Histopathol 1989;414(4):285-298

9 Weiss SW, Enzinger FM. Malignant fibrous histiocytoma: an analysis of 200 cases. Cancer 1978;41:2250-2266 